\title{
Brain MRI analysis for Alzheimer's disease diagnosis using an ensemble system of deep convolutional neural networks
}

\author{
Jyoti Islam* ${ }^{*}$ and Yanqing Zhang
}

\begin{abstract}
Alzheimer's disease is an incurable, progressive neurologicalbrain disorder. Earlier detection of Alzheimer's disease can help with proper treatment and prevent brain tissue damage. Several statistical and machine learning models have been exploited by researchers for Alzheimer's disease diagnosis. Analyzing magnetic resonance imaging (MRI) is a common practice for Alzheimer's disease diagnosis in clinical research. Detection of Alzheimer's disease is exacting due to the similarity in Alzheimer's disease MRI data and standard healthy MRI data of older people. Recently, advanced deep learning techniques have successfully demonstrated human-level performance in numerous fields including medical image analysis. We propose a deep convolutional neural network for Alzheimer's disease diagnosis using brainMRI data analysis. While most of the existing approaches perform binary classification, our model can identify different stages of Alzheimer's disease and obtains superior performance for early-stage diagnosis. We conducted ample experiments to demonstrate that our proposed model outperformed comparative baselines on the Open Access Series of Imaging Studies dataset.
\end{abstract}

Keywords: Neurological disorder, Alzheimer's disease, Deep learning, Convolutional neural network, MRI, Brain imaging

\section{Background}

Alzheimer's disease $(\mathrm{AD})$ is the most prevailing type of dementia. The prevalence of $\mathrm{AD}$ is estimated to be around 5\% after 65 years old and is staggering 30\% for more than 85 years old in developed countries. It is estimated that by 2050, around 0.64 Billion people will be diagnosed with $\mathrm{AD}$ [1]. Alzheimer's disease destroys brain cells causing people to lose their memory, mental functions and ability to continue daily activities. Initially, Alzheimer's disease affects the part of the brain that controls language and memory. As a result, AD patients suffer from memory loss, confusion and difficulty in speaking, reading or writing. They often forget about their life and may not recognize their family members. They struggle to perform daily activities such as brushing hair or combing tooth. All these make AD patients

*Correspondence: jislam2@student.gsu.edu

Department of Computer Science, Georgia State University, Atlanta, GA 30302-5060, USA anxious or aggressive or to wander away from home. Ultimately, AD destroys the part of the brain controlling breathing and heart functionality which lead to death.

There are three major stages in Alzheimer's diseasevery mild, mild and moderate. Detection of Alzheimer's disease (AD) is still not accurate until a patient reaches moderate AD stage. For proper medical assessment of $\mathrm{AD}$, several things are needed such as physical and neurobiological examinations, Mini-Mental State Examination (MMSE) and patient's detailed history. Recently, physicians are using brain MRI for Alzheimer's disease diagnosis. AD shrinks the hippocampus and cerebral cortex of the brain and enlarges the ventricles [2]. Hippocampus is the responsible part of the brain for episodic and spatial memory. It also works as a relay structure between our body and brain. The reduction in hippocampus causes cell loss and damage specifically to synapses and neuron ends. So neurons cannot communicate anymore via synapses. As a result, brain regions related to remembering (short-term memory), thinking, planning 
and judgment are affected [2]. The degenerated brain cells have low intensity in MRI images [3]. Figure 1 shows some brain MRI images with four different AD stages.

For accurate disease diagnosis, researchers have developed several computer-aided diagnostic systems. They developed rule-based expert systems from 1970s to 1990s and supervised models from 1990s [4]. Feature vectors are extracted from medical image data to train supervised systems. Extracting those features needs human experts that often require a lot of time, money and effort. With the advancement of deep learning models, now we can extract features directly from the images without the engagement of human experts. So researchers are focusing on developing deep learning models for accurate disease diagnosis. Deep learning technologies have achieved major triumph for different medical image analysis tasks such as MRI, microscopy, CT, ultrasound, X-ray and mammography. Deep models showed prominent results for organ and substructure segmentation, several disease detection and classification in areas of pathology, brain, lung, abdomen, cardiac, breast, bone, retina, etc. [4].

As the disease progresses, abnormal proteins (amyloid $\beta[\mathrm{A} \beta]$ and hyperphosphorylated tau) are accumulated in the brain of an AD patient. This abnormal protein accumulation leads to progressive synaptic, neuronal and axonal damage. The changes in the brain due to AD have a stereotypical pattern of early medial temporal lobe (entorhinal cortex and hippocampus) involvement, followed by progressive neocortical damage [5]. Such changes occur years before the AD symptoms appear. It looks like the toxic effects of hyperphosphorylated tau and/or amyloid $-\beta[\mathrm{A} \beta]$ which gradually erodes the brain, and when a clinical threshold is surpassed, amnestic symptoms start to develop. Structural MRI (sMRI) can be used for measuring these progressive changes in the brain due to the AD. Our research work focuses on analyzing sMRI data using deep learning model for Alzheimer's disease diagnosis.

Machine learning studies using neuroimaging data for developing diagnostic tools helped a lot for automated

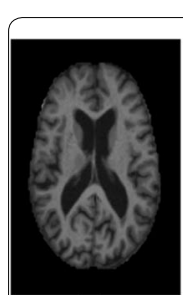

(a)

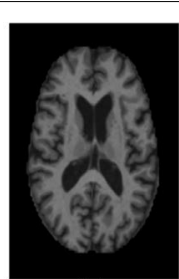

(b)

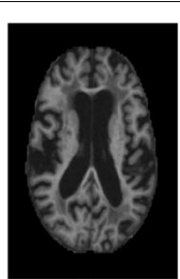

(c)

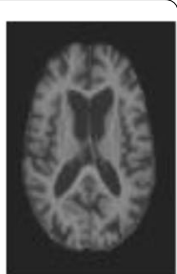

(d)
Fig. 1 Example of different brain MRI images presenting different Alzheimer's disease stages. a Non-demented; b Very mild dementia; c Mild dementia; d Moderate dementia brain MRI segmentation and classification. Most of them use handcrafted feature generation and extraction from the MRI data. These handcrafted features are fed into machine learning models such as support vector machine and logistic regression model for further analysis. Human experts play a crucial role in these complex multi-step architectures. Moreover, neuroimaging studies often have a dataset with limited samples. While image classification datasets used for object detection and classification have millions of images (for example, ImageNet database [6]), neuroimaging datasets usually contain a few hundred images. But a large dataset is vital to develop robust neural networks. Because of the scarcity of large image database, it is important to develop models that can learn useful features from the small dataset. Moreover, the state-of-the-art deep learning models are optimized to work with natural (every day) images. These models also require a lot of balanced training data to prevent overfitting in the network. We developed a deep convolutional neural network that learned features directly from the input sMRI and eliminated the need for the handcrafted feature generation. We trained our model using the OASIS database [7] that has only 416 sMRI data. Our proposed model can classify different stages of Alzheimer's disease and outperforms the offthe-shelf deep learning models. Hence, our primary contributions are threefold:

- We propose a deep convolutional neural network that can identify Alzheimer's disease and classify the current disease stage.

- Our proposed network learns from a small dataset and still demonstrates superior performance for $A D$ diagnosis.

- We present an efficient approach to training a deep learning model with an imbalanced dataset.

The rest of the paper is organized as follows. Section 2 discusses briefly about the related work on AD diagnosis. Section 3 presents the proposed model. Section 4 reports the experimental details and the results. Finally, in Sect. 5, we conclude the paper with our future research direction.

\section{Related work}

Detection of physical changes in brain complements clinical assessments and has an increasingly important role for early detection of AD. Researchers have been devoting their efforts to neuroimaging techniques to measure pathological brain changes related to Alzheimer's disease. Machine learning techniques have been developed to build classifiers using imaging data and clinical measures for AD diagnosis [8-17]. These studies have identified 
the significant structural differences in the regions such as the hippocampus and entorhinal cortex between the healthy brain and brain with AD. Changes in cerebrospinal tissues can explain the variations in the behavior of the AD patients $[18,19]$. Besides, there is a significant connection between the changes in brain tissues connectivity and behavior of AD patient [20]. The changes causing $\mathrm{AD}$ due to the degeneration of brain cells are noticeable on images from different imaging modalities, e.g., structural and functional magnetic resonance imaging (sMRI, fMRI), position emission tomography (PET), single photon emission computed tomography (SPECT) and diffusion tensor imaging (DTI) scans. Several researchers have used these neuroimaging techniques for AD Diagnosis. For example, sMRI [21-26], fMRI [27, 28], PET [29, 30], SPECT [31-33] and DTI [34, 35] have been used for diagnosis or prognosis of AD. Moreover, information from multiple modalities has been combined to improve the diagnosis performance [36-47].

A classic magnetic resonance imaging (MRI)-based automated AD diagnostic system has mainly two building blocks-feature/biomarker extraction from the MRI data and classifier based on those features/biomarkers. Though various types of feature extraction techniques exist, there are three major categories-(1) voxel-based approach, (2) region of interest (ROI)-based approach, and (3) patch-based approach. Voxel-based approaches are independent of any hypothesis on brain structures [48-51]. For example, voxel-based morphometry measures local tissue (i.e., white matter, gray matter and cerebrospinal fluid) density of the brain. Voxel-based approaches exploit the voxel intensities as the classification feature. The interpretation of the results is simple and intuitive in voxel-based representations, but they suffer from the overfitting problem since there are limited (e.g., tens or hundreds) subjects with very high (millions)dimensional features [52], which is a major challenge for $\mathrm{AD}$ diagnosis based on neuroimaging. To achieve more compact and useful features, dimensionality reduction is essential. Moreover, voxel-based approaches suffer from the ignorance of regional information.

Region of interest (ROI)-based approach utilizes the structurally or functionally predefined brain regions and extracts representative features from each region $[21,25,28,30,53-55]$. These studies are based on specific hypothesis on abnormal regions of the brain. For example, some studies have adopted gray matter volume [56], hippocampal volume [57-59] and cortical thickness $[21,60]$. ROI-based approaches are widely used due to relatively low feature dimensionality and whole brain coverage. But in ROI-based approaches, the extracted features are coarse as they cannot represent small or subtle changes related to brain diseases. The structural or functional changes that occur in the brain due to neurological disorder are typically spread to multiple regions of the brain. As the abnormal areas can be part of a single ROI or can span over multiple ROIs, voxel-based or ROI-based approaches may not efficiently capture the disease-related pathologies. Besides, the region of interest (ROI) definition requires expert human knowledge. Patch-based approaches [23, 61-66] divide the whole brain image into small-sized patches and extract feature vector from those patches. Patch extraction does not require ROI identification, so the necessity of human expert involvement is reduced compared to ROI-based approaches. Compared to voxel-based approaches, patch-based methods can capture the subtle brain changes with significantly reduced dimensionality. Patchbased approaches learn from the whole brain and better captures the disease-related pathologies that results in superior diagnosis performance. However, there is still challenges to select informative patches from the MRI images and generate discriminative features from those patches.

A large number of research works focused on developing advanced machine learning models for $\mathrm{AD}$ diagnosis using MRI data. Support vector machine SVM), logistic regressors (e.g., Lasso and Elastic Net), sparse representation-based classification (SRC), random forest classifier, etc., are some widely used approaches. For example, Kloppel et al. [50] used linear SVM to detect AD patients using T1 weighted MRI scan. Dimensional reduction and variations methods were used by Aversen [67] to analyze structural MRI data. They have used both SVM binary classifier and multi-class classifier to detect AD from MRI images. Vemuri et al. [68] used SVM to develop three separate classifiers with MRI, demographic and genotype data to classify AD and healthy patients. Gray [69] developed a multimodal classification model using random forest classifier for AD diagnosis from MRI and PET data. Er et al. [70] used gray-level co-occurrence matrix (GLCM) method for AD classification. Morra et al. [71] compared several model's performances for AD detection including hierarchical AdaBoost, SVM with manual feature and SVM with automated feature. For developing these classifiers, typically predefined features are extracted from the MRI data. However, training a classifier independent from the feature extraction process may result in sub-optimal performance due to the possible heterogeneous nature of the classifier and features [72].

Recently, deep learning models have been famous for their ability to learn feature representations from the input data. Deep learning networks use a layered, hierarchical structure to learn increasingly abstract feature representations from the data. Deep learning architectures learn simple, low-level features from the data and 
build complex high-level features in a hierarchy fashion. Deep learning technologies have demonstrated revolutionary performance in several areas, e.g., visual object recognition, human action recognition, natural language processing, object tracking, image restoration, denoising, segmentation tasks, audio classification and brain-computer interaction. In recent years, deep learning models specially convolutional neural network (CNN) have demonstrated excellent performance in the field of medical imaging, i.e., segmentation, detection, registration and classification [4]. For neuroimaging data, deep learning models can discover the latent or hidden representation and efficiently capture the disease-related pathologies. So, recently researchers have started using deep learning models for $\mathrm{AD}$ and other brain disease diagnosis.

Gupta et al. [62] have developed a sparse autoencoder model for $\mathrm{AD}$, mild cognitive impairment (MCI) and healthy control (HC) classification. Payan and Montana [65] trained sparse autoencoders and 3D CNN model for AD diagnosis. They also developed a 2D CNN model that demonstrated nearly identical performance. Brosch et al. [73] developed a deep belief network model and used manifold learning for AD detection from MRI images. Hosseini-Asl et al. [74] adapted a 3D CNN model for AD diagnostics. Liu and Shen [75] developed a deep learning model using both unsupervised and supervised techniques and classified $\mathrm{AD}$ and MCI patients. Liu et al. [76] have developed a multimodal stacked autoencoder network using zero-masking strategy. Their target was to prevent loss of any information of the image data. They have used SVM to classify the neuroimaging features obtained from MR/PET data. Sarraf and Tofighi [77] used fMRI data and deep LeNet model for AD detection. Suk et al. [23, 42, 78, 79] developed an autoencoder network-based model for AD diagnosis and used several complex SVM kernels for classification. They have extracted low- to mid-level features from magnetic current imaging (MCI), MCI-converter structural MRI, and PET data and performed classification using multikernel SVM. Cárdenas-Peña et al. [80] have developed a deep learning model using central kernel alignment and compared the supervised pre-training approach to two unsupervised initialization methods, autoencoders and principal component analysis (PCA). Their experiment shows that SAE with PCA outperforms three hidden layers SAE and achieves an increase of $16.2 \%$ in overall classification accuracy.

So far, AD is detected at a much later stage when treatment can only slow the progression of cognitive decline. No treatment can stop or reverse the progression of AD. So, early diagnosis of AD is essential for preventive and disease-modifying therapies. Most of the existing research work on AD diagnosis focused on binary classification problems, i.e., differentiating $\mathrm{AD}$ patients from healthy older adults. However, for early diagnosis, we need to distinguish among current AD stages, which makes it a multi-class classification problem. In our previous work [81], we developed a very deep convolutional network and classified the four different stages of the $\mathrm{AD}-$ non-demented, very mild dementia, mild dementia and moderate dementia. For our current work, we improved the previous model [81], developed an ensemble of deep convolutional neural networks and demonstrated better performance on the Open Access Series of Imaging Studies (OASIS) dataset [7].

\section{Methods}

\subsection{Formalization}

Let $x=\left\{x_{i}, i=1, \ldots, N\right\}$, a set of MRI data with $x_{i} \in[0,1,2, \ldots, L-1]^{h * w * l}$, a three-dimensional (3D) image with $L$ grayscale values, $h * w * l$ voxels and $y \in\{0,1,2,3\}$, one of the stages of AD where $0,1,2$ and 3 refer to non-demented, very mild dementia, mild dementia and moderate dementia, respectively. We will construct a classifier,

$$
f: X \rightarrow Y ; x \mapsto y,
$$

which predicts a label $y$ in response to an input image $x$ with minimum error rate. Mainly, we want to determine this classifier function $f$ by an optimal set of parameters $w \in \mathbb{R}^{P}$ (where $P$ can easily be in the tens of millions), which will minimize the loss or error rate of prediction. The training process of the classifier would be an iterative process to find the set of parameters $w$, which minimizes the classifier's loss

$$
L(w, X)=\frac{1}{n} \sum_{i=1}^{n} l\left(f\left(x_{i}, w\right), \widehat{c_{i}}\right)
$$

where $x_{i}$ is $i$ th image of $X, f\left(x_{i}, w\right)$ is the classifier function that predicts the class $c_{i}$ of $x_{i}$ given $w, \widehat{c_{i}}$ is the ground-truth class for $i$ th image $x_{i}$ and $l\left(c_{i}, \widehat{c_{i}}\right)$ is the penalty function for predicting $c_{i}$ instead of $\widehat{c_{i}}$. We set $l$ to the loss of cross-entropy,

$$
l=-\sum_{i} \widehat{c_{i}} \log c_{i}
$$

\subsection{Data selection}

In this study, we use the OASIS dataset [7] prepared by Dr. Randy Buckner from the Howard Hughes Medical Institute (HHMI) at Harvard University, the Neuroinformatics Research Group (NRG) at Washington University School of Medicine, and the Biomedical Informatics Research Network (BIRN). There are 416 subjects aged 
18-96, and for each of them, 3 or 4 T1-weighted sMRI scans are available. Hundred of the patients having age over 60 are included in the dataset with very mild to moderate $\mathrm{AD}$.

\subsection{Data augmentation}

Data augmentation refers to artificially enlarging the dataset using class-preserving perturbations of individual data to reduce the overfitting in neural network training [82]. The reproducible perturbations will enable new sample generation without changing the semantic meaning of the image. Since manually sourcing of additional labeled image is difficult in medical domain due to limited expert knowledge availability, data augmentation is a reliable way to increase the size of the dataset. For our work, we developed an augmentation scheme involving cropping for each image. We set the dimension of the crop similar to the dimension of the proposed deep CNN classifier. Then, we extracted three crops from each image, each for one of the image plane: axial or horizontal plane, coronal or frontal plane, and sagittal or median plane. For our work, we use $80 \%$ data from the OASIS dataset as training set and $20 \%$ as test dataset. From the training dataset, a random selection of $10 \%$ images is used as validation dataset. The augmentation process is performed separately for the train, validation and test dataset. One important thing to consider is the data augmentation process is different from classic crossvalidation scheme. Data augmentation is used to reduce overfitting in a vast neural network while training with a small dataset. On the other hand, cross-validation is used to derive a more accurate estimate of model prediction performance. Cross-validation technique is computationally expensive for a deep convolutional neural network training as it takes an extensive amount of time.

\subsection{Network architecture}

Our proposed network is an ensemble of three deep convolutional neural networks with slightly different configurations. We made a considerable amount of effort for the design of the proposed system and the choice of the architecture. All the individual models have a common architectural pattern consisted of four basic operations:

- convolution

- batch normalization [83]

- rectified linear unit, and

- pooling

Each of the individual convolutional neural networks has several layers performing these four basic operations illustrated in Fig. 2. The layers in the model follow a particular connection pattern known as dense connectivity [84] as

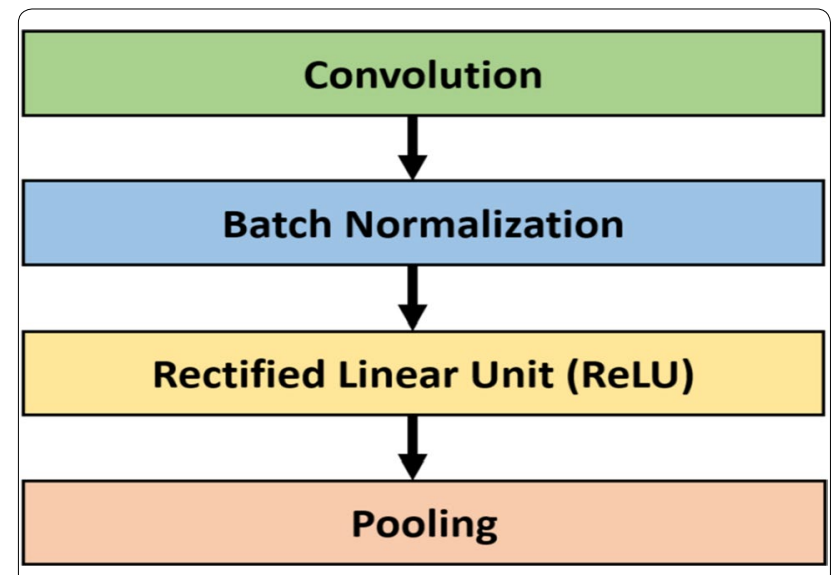

Fig. 2 Common building block of the proposed ensemble model

shown in Fig. 3. The dense connections have a regularizing effect that reduces overfitting in the network while training with a small dataset. We keep these layers very narrow (e.g., 12 filters per layer) and connect each layer to every other layer. Similar to [84], we will refer to the layers as dense layer and combination of the layers as dense block. Since all the dense layers are connected to each other, the $i$ th layer receives the feature maps $\left(h_{0}, h_{1}, h_{2}, \ldots, h_{i-1}\right)$, from all previous layers $(0,1,2, \ldots, i-1)$. Consequently, the network has a global feature map set, where each layer adds a small set of feature maps. In times of training, each layer can access the gradients from the loss function as well as the original input. Therefore, the flow of information improves, and gradient flow becomes stronger in the network. Figure 4 shows the intermediate connection between two dense blocks.

For the design of the proposed system, we experimented with several different deep learning architectures and finally developed an ensemble of three homogeneous deep convolution neural networks. The proposed model is shown in Fig. 5. We will refer to the individual models as $M_{1}, M_{2}$ and $M_{3}$. In Fig. 5, the top network is $M_{1}$, the middle network is $M_{2}$, and the bottom network is $M_{3}$. Each of the models consists of several convolution layers, pooling layers, dense blocks and transition layers. The transition layer is a combination of batch normalization layer, a $1^{*} 1$ convolutional layer followed by a $2 * 2$ average pooling layer with stride 2. Batch normalization [83] acts as a regularizer and speeds up the training process dramatically. Traditional normalization process (shifting inputs to zero-mean and unit variance) is used as a preprocessing step. Normalization is applied to make the data comparable across features. When the data flow inside the network at the time of training process, the weights and parameters are continuously adjusted. Sometimes these adjustments make the data too big or too small, 


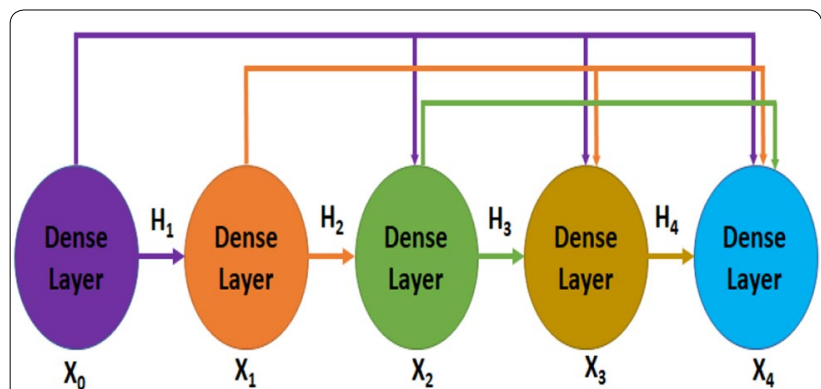

Fig. 3 Illustration of dense connectivity with a 5-layer dense block

a problem referred as 'Internal Covariance Shift.' Batch normalization largely eliminates this problem. Instead of doing the normalization at the beginning, batch normalization is performed to each mini-batches along with SGD training. If $\mathfrak{B}=\left\{x_{1}, x_{2}, \ldots, x_{m}\right\}$ is a minibatch of $m$ activations value, the normalized values are $\left(\widehat{x}_{1}, \widehat{x}_{2}, \ldots, \widehat{x}_{m}\right)$ and the linear transformations are $y_{1}, y_{2}, \ldots, y_{m}$, then batch normalization is referred to the transform:

$$
B N_{\gamma, \beta}: x_{1}, x_{2}, \ldots, x_{m} \rightarrow y_{1}, y_{2}, \ldots, y_{m}
$$

Considering $\gamma, \beta$ the parameters to be learned and $\epsilon$, a constant added to the mini-batch variance for numerical stability, batch normalization is given by the following equations:

$$
\mu_{\mathfrak{B}} \leftarrow \frac{1}{m} \sum_{i=1}^{m} x_{i}
$$

$$
\begin{aligned}
& \sigma_{\mathfrak{B}}^{2} \leftarrow \frac{1}{m} \sum_{i=1}^{m}\left(x_{i}-\mu_{\mathfrak{B}}\right)^{2} \\
& \widehat{x}_{i} \leftarrow \frac{x_{i}-\mu_{\mathfrak{B}}}{\sqrt{\sigma_{\mathfrak{B}}^{2}+\epsilon}} \\
& y_{i} \leftarrow \gamma \widehat{x_{i}}+\beta \equiv B N_{\gamma, \beta}\left(x_{i}\right)
\end{aligned}
$$

where $\mu_{\mathfrak{B}}$ is mini-batch mean and $\sigma_{\mathfrak{B}}^{2}$ is mini-batch variance [83].

Though each model has four dense blocks, they differ in the number of their internal $1 * 1$ convolution and $3 * 3$ convolution layers. The first model, $\mathrm{M}_{1}$, has six $(1 * 1 \mathrm{con}$ volution and $3 * 3$ convolution layers) in the first dense block, twelve ( $1 * 1$ convolution and $3 * 3$ convolution layers) in the second dense block, twenty-four ( $1 * 1$ convolution and $3 * 3$ convolution layers) in the third dense block and sixteen ( $1 * 1$ convolution and $3 * 3$ convolution layers) in the fourth dense block. The second model, $\mathrm{M}_{2}$, and third model, $M_{3}$, have $(6,12,32,32)$ and $(6,12,36,24)$ arrangement respectively. Because of the dense connectivity, each layer has direct connections to all subsequent layers, and they receive the feature maps from all preceding layers. So, the feature maps work as global state of the network, where each layer can add their own feature map. The global state can be accessed from any part

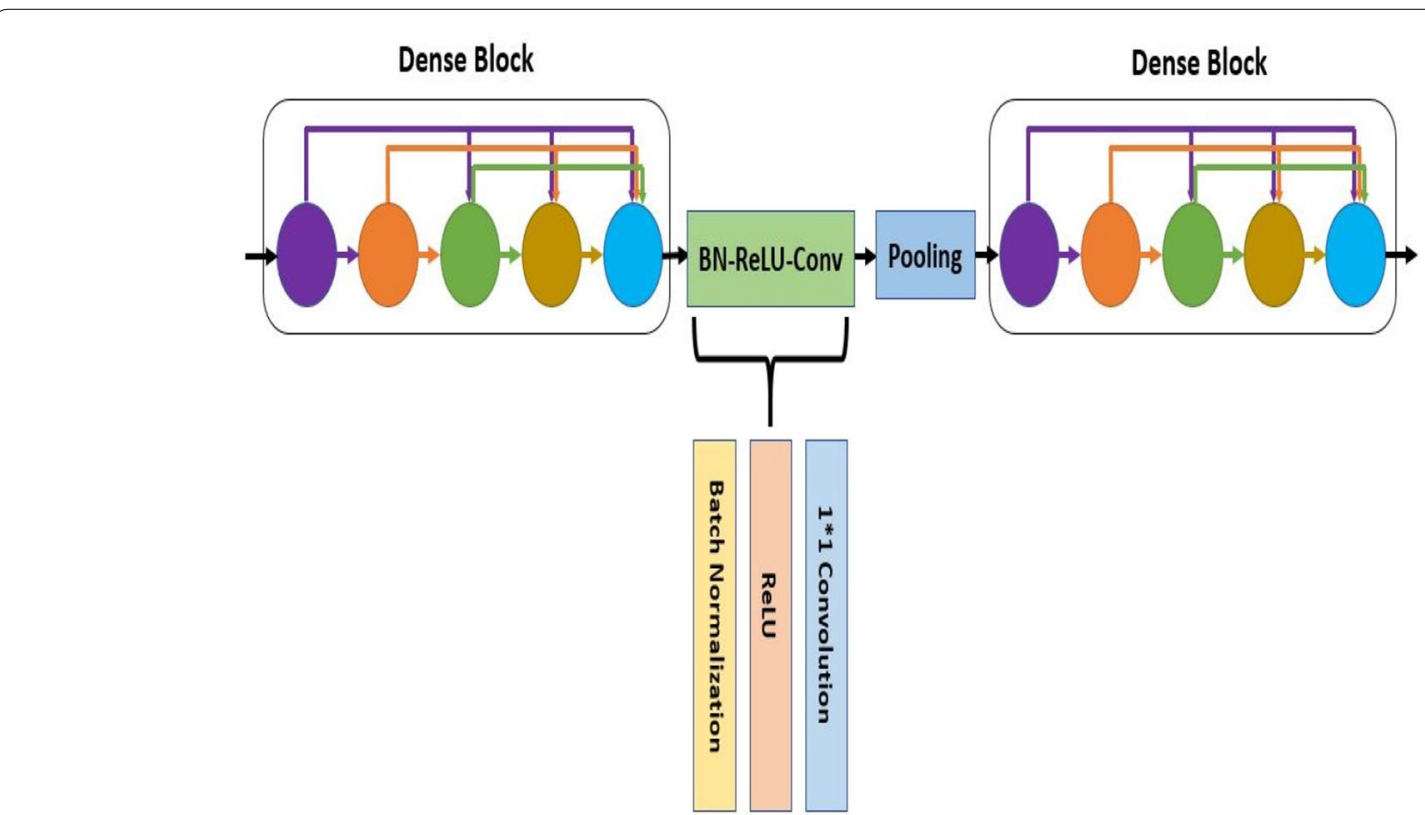

Fig. 4 Illustration of two dense blocks and their intermediate connection 


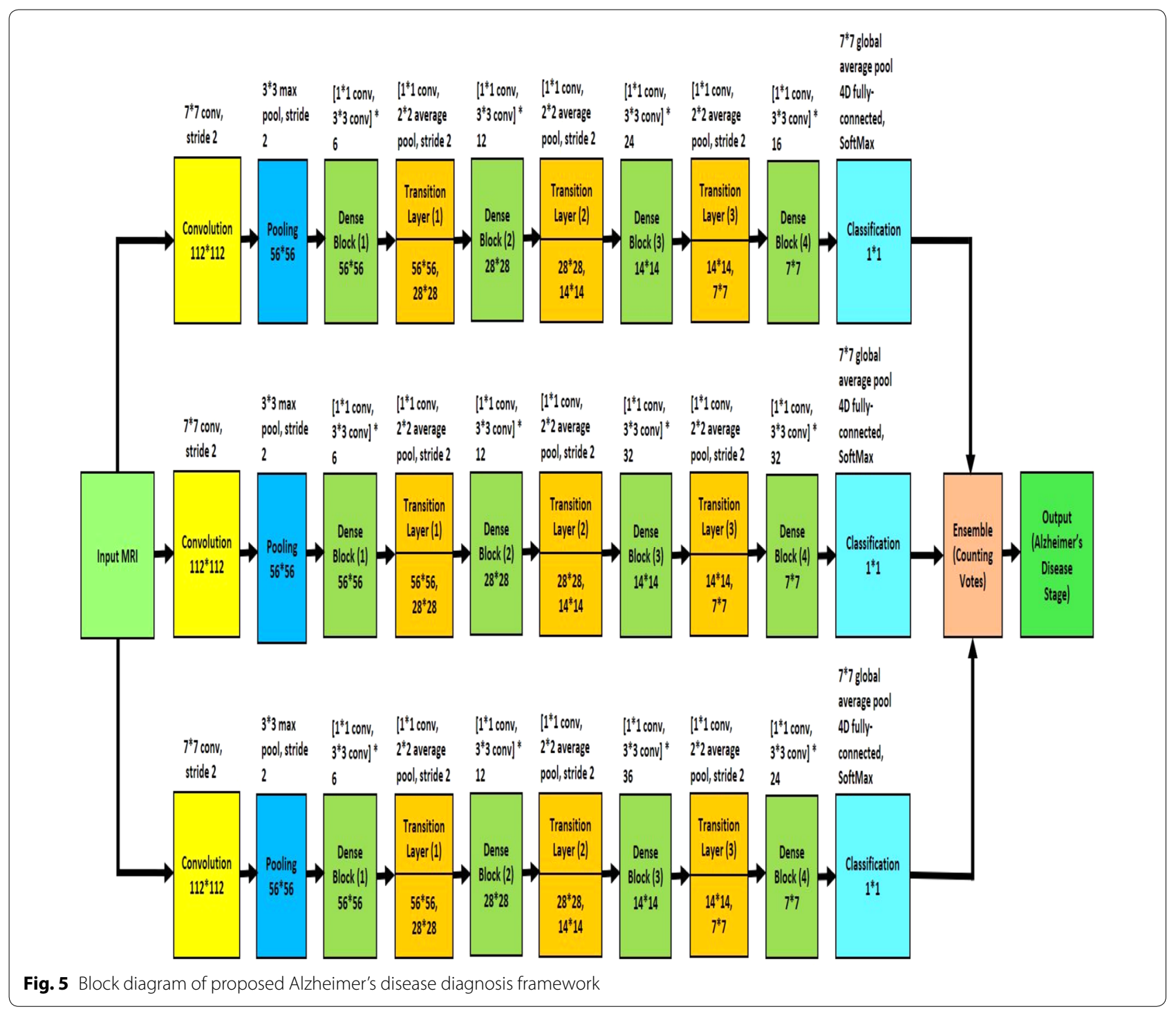

of the network and how much each layer can contribute to is decided by the growth rate of the network. Since the feature maps of different layers are concatenated together, the variation in the input of subsequent layers increases and results in more efficiency.

The input MRI is 3D data, and our proposed model is a $2 \mathrm{D}$ architecture, so we devise an approach to convert the input data to 2D images. For each MRI data, we created patches from three physical planes of imaging: axial or horizontal plane, coronal or frontal plane, and sagittal or median plane. These patches are fed to the proposed network as input. Besides, this data augmentation technique increases the number of samples in training dataset. The size of each patch is $112 * 112$. We trained the individual models separately, and each of them has own softmax layer for classification decision.
The softmax layers have four different output classes: non-demented, very mild, mild and moderate AD. The individual models take the input image and generate its learned representation. The input image is classified to any of the four output classes based on this feature representation. To measure the loss of each of these models, we used cross-entropy. The softmax layer takes the learned representation, $f_{i}$, and interprets it to the output class. A probability score, $p_{i}$, is also assigned for the output class. If we define the number of output classes as $\mathrm{m}$, then we get

$$
p_{i}=\frac{\exp \left(f_{i}\right)}{\sum_{i} \exp \left(f_{i}\right)}, i=1, \ldots, m
$$


and

$$
L=-\sum_{i} t_{i} \log \left(p_{i}\right)
$$

where $\mathrm{L}$ is the loss of cross-entropy of the network. Backpropagation is used to calculate the gradients of the network. If the ground truth of an MRI data is denoted as $t_{i}$, then

$$
\frac{\partial L}{\partial f_{i}}=p_{i}-t_{i}
$$

To handle the imbalance in the dataset, we used cost-sensitive training [85]. A cost matrix $\xi$ was used to modify the output of the last layer of the individual networks. Since the less frequent classes (very mild dementia, mild dementia, moderate dementia) are underrepresented in the training dataset, the output of the networks was modified using the cost matrix $\xi$ to give more importance to these classes. If $o$ is the output of the individual model, $p$ is the desired class and $L$ is the loss function, then $y$ denotes the modified output:

$$
y^{i}=L\left(\xi_{p}, o^{i}\right),: \quad y_{p}^{i} \geq y_{j}^{i} \quad \forall j \neq p
$$

The loss function is modified as:

$$
L=-\sum_{n} t_{n} \log \left(y_{n}\right)
$$

where $y_{n}$ incorporates the class-dependent cost $\xi$ and is related to the output $o_{n}$ via the softmax function [85]:

$$
y_{n}=\frac{\xi_{p, n} \exp \left(o_{n}\right)}{\sum_{k} \xi_{p, k} \exp \left(o_{k}\right)}
$$

The weight of a particular class is dependent on the number of samples of that class. If class $r$ has $q$ times more samples than those of $s$, the target is to make one sample of class $s$ to be as important as $q$ samples of class $r$. So, the class weight of $s$ would be $q$ times more than the class weight of $r$.

We optimized the individual models with the stochastic gradient descent (SGD) algorithm. For regularization, we used early stopping. We split the training dataset into a training set and a cross-validation set in 9:1 proportion. Let $L_{t r}(t)$ and $L_{v a}(t)$ are the average error per example over the training set and validation set respectively, measured after $t$ epoch. Training was stopped as soon as it reached convergence, i.e., validation error $L_{v a}(t)$ does not improve for $t$ epoch and $L_{v a}(t)>L_{v a}(t-1)$. We used Nesterov momentum optimization with Stochastic Gradient Descent (SGD) algorithm for minimizing the loss of the network. Given an objective function $f(\theta)$ to be minimized, classic momentum is given by the following pair of equations:

$$
\begin{aligned}
& v_{t}=\mu v_{t-1}-\epsilon \nabla f\left(\theta_{t-1}\right) \\
& \theta_{t}=\theta_{t-1}+v_{t}
\end{aligned}
$$

where $v_{t}$ refers to the velocity, $\epsilon>0$ is the learning rate, $\mu \in[0,1]$ is the momentum coefficient and $\nabla f \theta_{t}$ is the gradient at $\theta_{t}$. On the other hand, Nesterov momentum is given by:

$$
\begin{aligned}
& v_{t}=\mu v_{t-1}-\epsilon \nabla f\left(\theta_{t-1}+\mu v_{t-1}\right) \\
& \theta_{t}=\theta_{t-1}+v_{t}
\end{aligned}
$$

The output classification labels of the three individual model are ensembled together using majority voting technique. Each classifier 'votes' for a particular class, and the class with the majority votes would be assigned as the label for the input MRI data.

\section{Results and discussion}

\subsection{Experimental settings}

We implemented the proposed model using Tensorflow [86], Keras[87] and Python on a Linux X86-64 machine with AMD A8 CPU, 16 GB RAM and NVIDIA GeForce GTX 770. We applied the SGD training with a mini-batch size of 64 , a learning rate of 0.01 , a weight decay of 0.06 and a momentum factor of 0.9 with Nesterov optimization. We applied early stopping in the SGD training process, while there was no improvement (change of less than 0.0001) in validation loss for last six epoch.

To validate the effectiveness of the proposed AD detection and classification model, we developed two baseline deep CNN, Inception-v4 [88] and ResNet [89] and modified their architecture two classify 3D brain MRI data. Besides, we developed two different models, $M_{4}$ and $M_{5}$ having similar architecture like $M_{1}, M_{2}$ and $M_{3}$ model except for the number of layers in the dense block. $\mathrm{M}_{4}$ has six (1*1 convolution and $3 * 3$ convolution layers) in the first dense block, twelve (1*1 convolution and $3 * 3$ convolution layers) in the second dense block, forty-eight (1*1 convolution and 3*3 convolution layers) in the third dense block and thirty-two (1*1 convolution and $3 * 3$ convolution layers) in the fourth dense block (Fig. 6). The layers in the dense blocks of $M_{5}$ have the arrangement 6,12 , 64, 48 as shown in Fig. 7. Additionally, we implemented two variants of our proposed model using $M_{4}$ and $M_{5}$. 


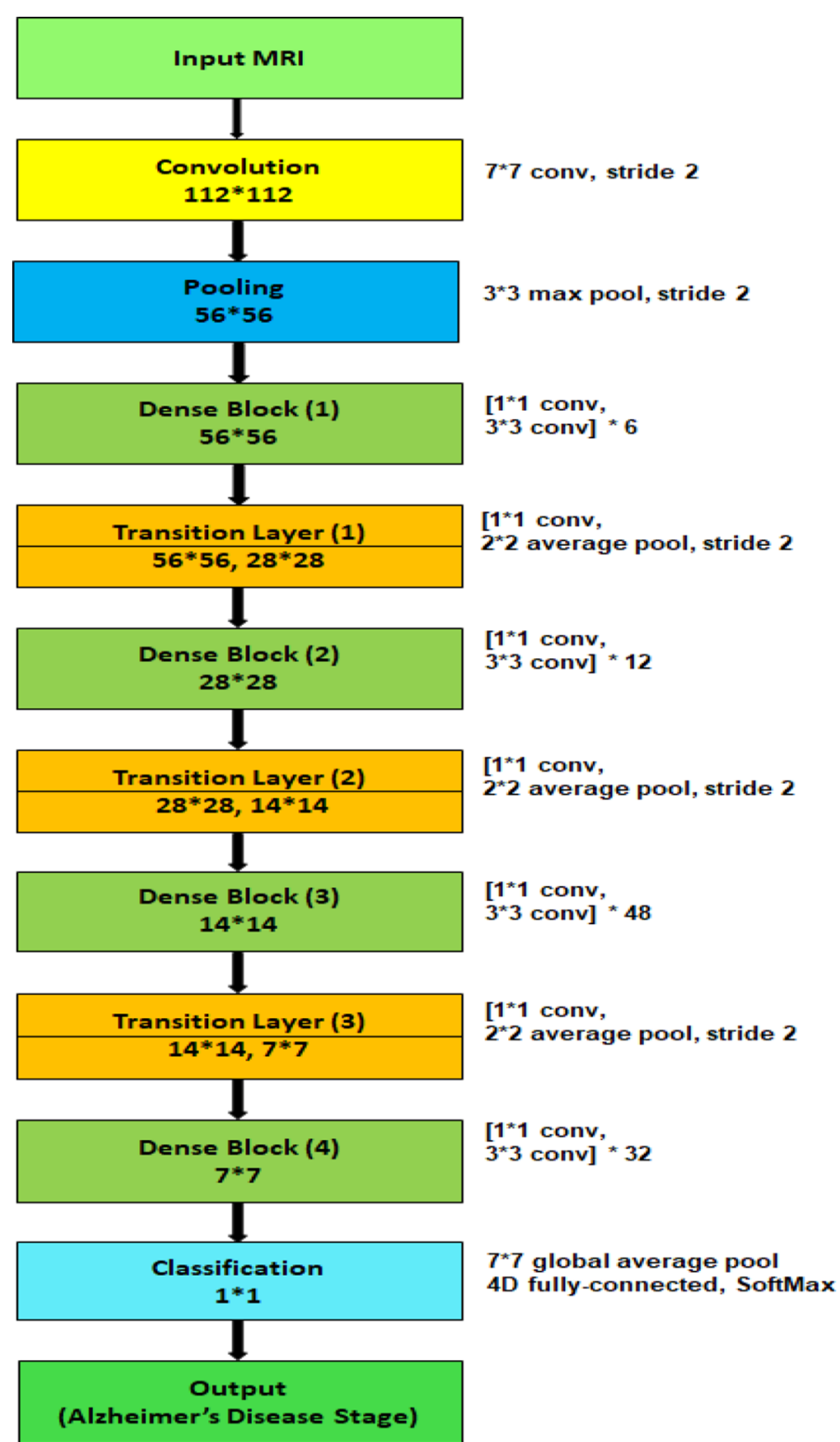

Fig. 6 Block diagram of individual model $M_{4}$

- For the first variant, we implemented an ensemble of four deep convolutional neural networks: $\mathrm{M}_{1}, \mathrm{M}_{2}, \mathrm{M}_{3}$ and $\mathrm{M}_{4}$. We will refer to this model as $\mathrm{E}_{1}$.

- For the second variant, we implemented an ensemble system of five deep convolutional neural networks: $M_{1}, M_{2}, M_{3}, M_{4}$ and $M_{5}$. We will refer to this model as $\mathrm{E}_{2}$.

\subsection{Performance metric}

Four metrics are used for quantitative evaluation and comparison, including accuracy, positive predictive value (PPV) or precision, sensitivity or recall, and the harmonic mean of precision and sensitivity (f1-score).
We denote TP, TN, FP and FN as true positive, true negative, false positive and false negative, respectively. The evaluation metrics are defined as:

$$
\begin{aligned}
\text { accuracy } & =\frac{(\mathrm{TP}+\mathrm{TN})}{(\mathrm{TP}+\mathrm{FP}+\mathrm{FN}+\mathrm{TN})} \\
\text { precision } & =\frac{\mathrm{TP}}{(\mathrm{TP}+\mathrm{FP})} \\
\text { recall } & =\frac{\mathrm{TP}}{(\mathrm{TP}+\mathrm{FN})} \\
f 1 \text {-score } & =\frac{(2 \mathrm{TP})}{(2 \mathrm{TP}+\mathrm{FP}+\mathrm{FN})}
\end{aligned}
$$




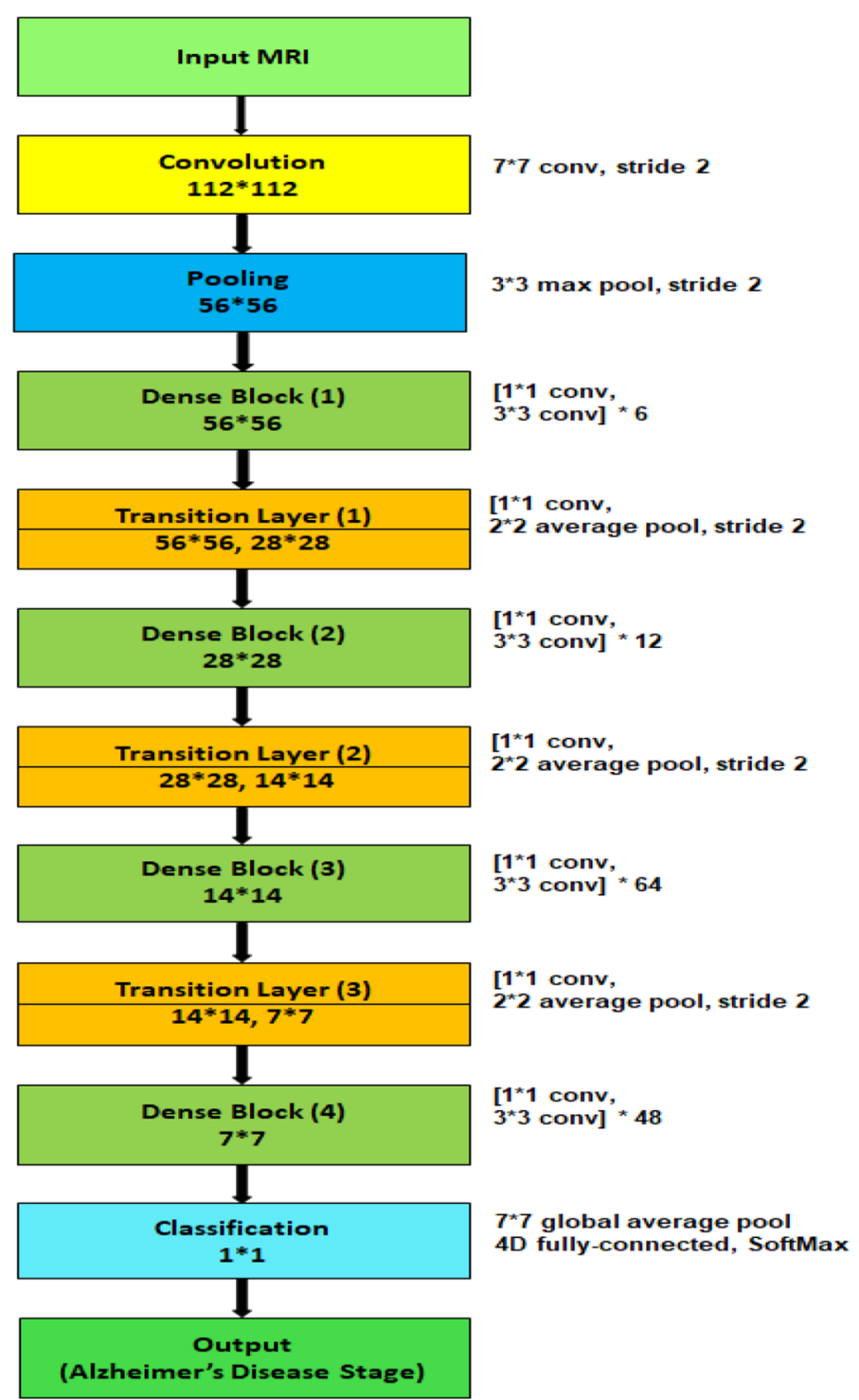

Fig. 7 Block diagram of individual model $M_{5}$

\subsection{Dataset}

The OASIS dataset [7] has 416 data samples. The dataset is divided into a training dataset and a test dataset in 4:1

Table 1 Classification performance of $\mathrm{M}_{1}$ model

\begin{tabular}{llllc}
\hline Class & Precision & Recall & f1-score & Support \\
\hline Non-demented & 0.99 & 0.99 & 0.99 & 73 \\
Very mild & 0.75 & 0.50 & 0.60 & 6 \\
Mild & 0.62 & 0.71 & 0.67 & 7 \\
Moderate & 0.33 & 0.50 & 0.40 & 2 \\
Avg/total & 0.93 & 0.92 & 0.92 & 88 \\
\hline
\end{tabular}

proportion. A validation dataset was prepared using $10 \%$ data from the training dataset.

\subsection{Results}

We report the classification performance of $M_{1}, M_{2}, M_{3}$, $\mathrm{M}_{4}$ and $\mathrm{M}_{5}$ model in Tables 1, 2, 3, 4 and 5, respectively. From the results, we notice that $M_{1}, M_{2}$ and $M_{3}$ model are the top performers among all models. So, we choose the ensemble of $M_{1}, M_{2}, M_{3}$ for our final architecture. Besides, the variants $E_{1}\left(M_{1}+M_{2}+M_{3}+M_{4}\right)$ and $E_{2}$ $\left(M_{1}+M_{2}+M_{3}+M_{4}+M_{5}\right)$ demonstrate inferior performance compared to the ensemble of $\mathrm{M}_{1}, \mathrm{M}_{2}, \mathrm{M}_{3}$ (proposed model) as shown in Fig. 8. From Fig. 8, we notice 
Table 2 Classification performance of $\mathrm{M}_{2}$ model

\begin{tabular}{lllll}
\hline Class & Precision & Recall & f1-score & Support \\
\hline Non-demented & 0.88 & 0.95 & 0.91 & 73 \\
Very mild & 0.00 & 0.00 & 0.00 & 6 \\
Mild & 0.25 & 0.29 & 0.27 & 7 \\
Moderate & 0.00 & 0.00 & 0.00 & 2 \\
Avg/total & 0.75 & 0.81 & 0.78 & 88 \\
\hline
\end{tabular}

Table 3 Classification performance of $\mathrm{M}_{3}$ model

\begin{tabular}{lllll}
\hline Class & Precision & Recall & f1-score & Support \\
\hline Non-demented & 0.99 & 0.96 & 0.97 & 73 \\
Very mild & 0.50 & 0.33 & 0.40 & 6 \\
Mild & 0.45 & 0.71 & 0.56 & 7 \\
Moderate & 0.50 & 0.50 & 0.50 & 2 \\
Avg/total & 0.90 & 0.89 & 0.89 & 88 \\
\hline
\end{tabular}

Table 4 Classification performance of $\mathrm{M}_{4}$ model

\begin{tabular}{llllc}
\hline Class & Precision & Recall & f1-score & Support \\
\hline Non-Demented & 0.92 & 0.67 & 0.77 & 73 \\
Very Mild & 0.00 & 0.00 & 0.00 & 6 \\
Mild & 0.17 & 0.60 & 0.26 & 7 \\
Moderate & 0.00 & 0.00 & 0.00 & 2 \\
Avg/Total & 0.77 & 0.61 & 0.66 & 88 \\
\hline
\end{tabular}

Table 5 Classification performance of $\mathrm{M}_{5}$ model

\begin{tabular}{llllc}
\hline Class & Precision & Recall & f1-score & Support \\
\hline Non-demented & 0.80 & 0.94 & 0.86 & 73 \\
Very Mild & 0.00 & 0.00 & 0.00 & 6 \\
Mild & 0.22 & 0.14 & 0.17 & 7 \\
Moderate & 0.00 & 0.00 & 0.00 & 2 \\
Avg/Total & 0.64 & 0.74 & 0.68 & 88 \\
\hline
\end{tabular}

Table 6 Performance of the proposed ensembled model

\begin{tabular}{lllll}
\hline Class & Precision & Recall & f1-score & Support \\
\hline Non-demented & 0.97 & 1.00 & 0.99 & 73 \\
Very mild & 1.00 & 0.33 & 0.50 & 6 \\
Mild & 0.67 & 0.86 & 0.75 & 7 \\
Moderate & 0.50 & 0.50 & 0.50 & 2 \\
Avg/total & 0.94 & 0.93 & 0.92 & 88 \\
\hline
\end{tabular}

that $\mathrm{E}_{1}$ model has an accuracy of $78 \%$ with $68 \%$ precision, $78 \%$ recall and $72 \% \mathrm{f} 1$ score. On the other hand, the $\mathrm{E}_{2}$ model demonstrates $77 \%$ accuracy with $73 \%$ precision, $77 \%$ recall and $75 \% \mathrm{f} 1$-score.

Table 6 shows the per-class classification performance of our proposed ensembled model on the OASIS dataset [7]. The accuracy of the proposed model is $93.18 \%$ with $94 \%$ precision, $93 \%$ recall and $92 \%$ f1-score. The performance comparison of classification results of the proposed ensembles model, and the two baseline deep CNN models are presented in Fig. 9. Inception-v4 [88] and ResNet [89] have demonstrated outstanding performance for object detection and classification. The reason behind their poor performance for $\mathrm{AD}$ detection and classification can be explained by the lack of enough training dataset.

Since these two networks are very deep neural networks, so without a large dataset, training process would not work correctly. On the other hand, the depth of our model is relatively low, and all the layers are connected to all preceding layers. So, there is a strong gradient flow in times of training that eliminates the 'Vanishing gradient' problem. In each training iteration, all the weights of a neural network receive an update proportional to the gradient of the error function concerning the current weight. But in some cases, the gradient will be vanishingly small and consequently prevent the weight from changing its value. It may completely stop the neural network from further training in worst-case scenario. Our proposed model does not suffer this 'Vanishing gradient' problem, have better feature propagation and provides better classification result even for the small dataset. The performance comparison of classification results of the proposed ensembled model, the baseline deep CNN models and the most recent work, ADNet [81] is presented in Fig. 10. It can be observed that proposed ensembled model achieves encouraging performance and outperforms the other models.

\section{Conclusion}

We made an efficient approach to AD diagnosis using brain MRI data analysis. While the majority of the existing research works focuses on binary classification, our model provides significant improvement for multi-class classification. Our proposed network can be very beneficial for early-stage AD diagnosis. Though the proposed model has been tested only on AD dataset, we believe it can be used successfully for other classification problems of medical domain. Moreover, the 


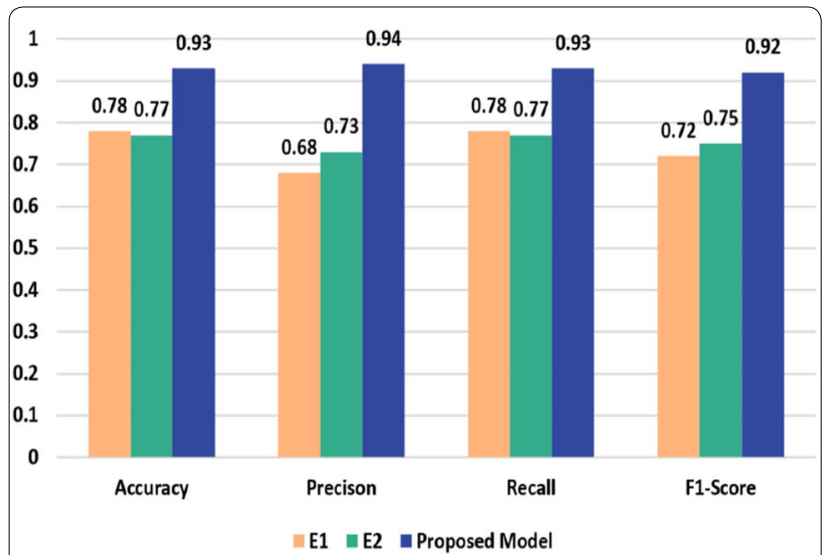

Fig. 8 Performance comparison of the proposed model and the variants

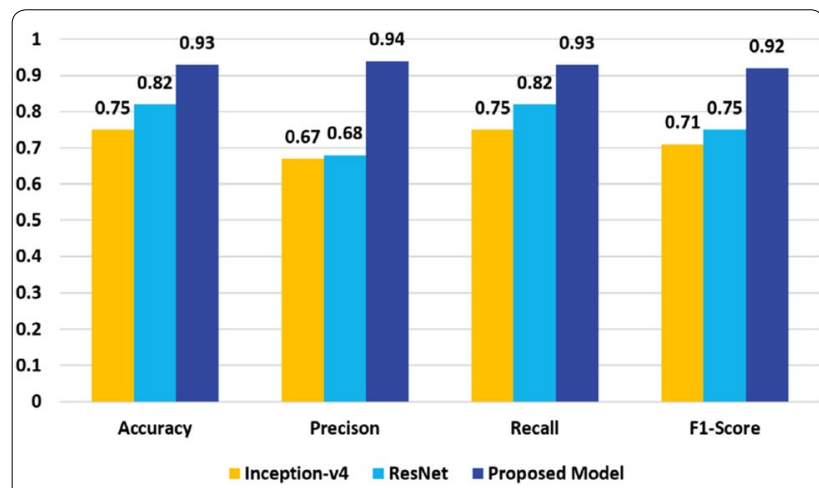

Fig. 9 Performance comparison of the proposed model and the baseline deep CNNs

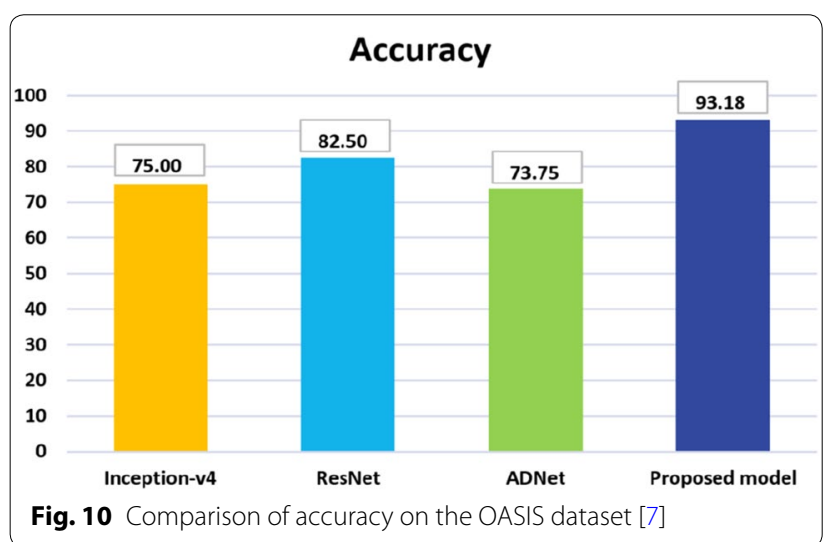

proposed approach has strong potential to be used for applying CNN into other areas with a limited dataset. In future, we plan to evaluate the proposed model for different $\mathrm{AD}$ datasets and other brain disease diagnosis.

\section{Authors' contributions}

Il carried out the background study, proposed the ensembled deep convolutional neural network, implemented the network, evaluated the result and drafted the manuscript. YZ supervised the work, proposed the variants of the models, monitored result evaluation process, and drafted the manuscript. Both authors read and approved the final manuscript.

\section{Authors' information}

Jyoti Islam is a PhD student at the Department of Computer Science, Georgia State University, Atlanta, GA, USA. Before joining GSU, she was a Senior Software Engineer at Samsung R\&D Institute Bangladesh. She received her M.Sc. degree in Computer Science and Engineering from University of Dhaka, Bangladesh, in 2012 under the supervision of Dr. Saifuddin Md. Tareeq. She received her B.Sc. degree in Computer Science and Engineering from the University of Dhaka, Bangladesh, in 2010. Her research is focused on deep learning and in particular in the area of medical image analysis for neurological disorder diagnosis. Her research interest extends to machine learning, computer vision, health informatics and software engineering.

Yanqing Zhang is currently a full Professor at the Computer Science Department at Georgia State University, Atlanta, GA, USA. He received the Ph.D. degree in computer science from the University of South Florida in 1997. His research areas include computational intelligence, data mining, deep learning, machine learning, bioinformatics, web intelligence, and intelligent parallel/distributed computing. He mainly focuses on research in computational intelligence (neural networks, fuzzy logic, evolutionary computation, kernel machines, and swarm intelligence). He has co-authored two books, co-edited two books and four conference proceedings. He has published 18 book chapters, 78 journal papers and 164 conference/workshop papers. He has served as a reviewer for over 70 international journals and as a program committee member for over 150 international conferences and workshops. He was Program Co-Chair: the 2013 IEEE/ACM/WIC International Conference on Web Intelligence, and the 2009 International Symposium on Bioinformatics Research and Applications. He was Program Co-Chair and Bioinformatics Track Chair of IEEE 7th International Conference on Bioinformatics \& Bioengineering in 2007, and Program Co-Chair of the 2006 IEEE International Conference on Granular Computing.

\section{Acknowledgements}

This study was supported by Brains and Behavior (B\&B) Fellowship program from Neuroscience Institute of Georgia State University. Data were provided by the Open Access Series of Imaging Studies [OASIS: Longitudinal: Principal Investigators: D. Marcus, R, Buckner, J. Csernansky, J. Morris; P50 AG05681, P01 AG03991, P01 AG026276, R01 AG021910, P20 MH071616, U24 RR021382]

\section{Competing interests}

The authors declare that they have no competing interests.

\section{Ethics approval and consent to participate}

Not applicable.

\section{Publisher's Note}

Springer Nature remains neutral with regard to jurisdictional claims in published maps and institutional affiliations.

Received: 12 February 2018 Accepted: 18 April 2018

Published online: 31 May 2018

\section{References}

1. Brookmeyer R, Johnson E, Ziegler-Graham K, Arrighi HM (2007) Forecasting the global burden of Alzheimer's disease. Alzheimer's dement 3(3):186-191

2. Sarraf S, Anderson J, Tofighi G (2016) Deepad: Alzheimer's disease classification via deep convolutional neural networks using MRI and fMRI. bioRxiv p 070441

3. Warsi MA (2012) The fractal nature and functional connectivity of brain function as measured by BOLD MRI in Alzheimer's disease. Ph.D. thesis 
4. Litjens G, Kooi T, Bejnordi BE, Setio AAA, Ciompi F, Ghafoorian M, van der Laak JA, van Ginneken B, Sánchez CI (2017) A survey on deep learning in medical image analysis. arXiv:1702.05747

5. Frisoni G, Fox NC, Jack C, Scheltens P, Thompson P (2010) The clinical use of structural MRI in Alzheimer's disease. Nat Rev Neurol 6:67-77

6. Russakovsky O, Deng J, Su H, Krause J, Satheesh S, Ma S, Huang Z, Karpathy A, Khosla A, Bernstein M, Berg AC, Fei-Fei L (2015) Imagenet large scale visual recognition challenge. Int J Comput Vis IJCV 115(3):211-252

7. Marcus DS, Wang TH, Parker J, Csernansky JG, Morris JC, Buckner RL (2007) Open access series of imaging studies (OASIS): cross-sectional MRI data in young, middle aged, nondemented, and demented older adults. J Cogn Neurosci 19(9):1498-1507

8. Davatzikos C, Fan Y, Wu X, Shen D, Resnick SM (2008) Detection of prodromal Alzheimer's disease via pattern classification of magnetic resonance imaging. Neurobiol Aging 29(4):514-523

9. Desikan RS, Cabral HJ, Settecase F, Hess CP, Dillon WP, Glastonbury CM, Weiner MW, Schmansky NJ, Salat DH, Fischl B (2010) Automated MRI measures predict progression to Alzheimer's disease. Neurobiol Aging 31(8):1364-1374

10. Fan Y, Batmanghelich N, Clark CM, Davatzikos C, Initiative ADN et al (2008) Spatial patterns of brain atrophy in MCl patients, identified via high-dimensional pattern classification, predict subsequent cognitive decline. Neuroimage 39(4):1731-1743

11. Fan Y, Resnick SM, Wu X, Davatzikos C (2008) Structural and functional biomarkers of prodromal Alzheimer's disease: a high-dimensional pattern classification study. Neuroimage 41(2):277-285

12. Filipovych R, Davatzikos C, Initiative ADN et al (2011) Semi-supervised pattern classification of medical images: application to mild cognitive impairment (MCI). Neurolmage 55(3):1109-1119

13. Hu K, Wang Y, Chen K, Hou L, Zhang X (2016) Multi-scale features extraction from baseline structure MRI for MCI patient classification and AD early diagnosis. Neurocomputing 175:132-145

14. Misra C, Fan Y, Davatzikos C (2009) Baseline and longitudinal patterns of brain atrophy in $\mathrm{MCl}$ patients, and their use in prediction of short-term conversion to ad: results from ADNI. Neuroimage 44(4):1415-1422

15. Moradi E, Pepe A, Gaser C, Huttunen H, Tohka J, Initiative ADN et al (2015) Machine learning framework for early MRI-based Alzheimer's conversion prediction in MCl subjects. Neuroimage 104:398-412

16. Rathore S, Habes M, Iftikhar MA, Shacklett A, Davatzikos C (2017) A review on neuroimaging-based classification studies and associated feature extraction methods for Alzheimer's disease and its prodromal stages. Neurolmage 155:530-548

17. de Vos F, Schouten TM, Hafkemeijer A, Dopper EG, van Swieten JC, de Rooij M, van der Grond J, Rombouts SA (2016) Combining multiple anatomical MRI measures improves Alzheimer's disease classification. Hum Brain Map 37(5):1920-1929

18. Fletcher E, Villeneuve S, Maillard P, Harvey D, Reed B, Jagust W, DeCarli C (2016) $\beta$-amyloid, hippocampal atrophy and their relation to longitudinal brain change in cognitively normal individuals. Neurobiol Aging 40:173-180

19. Serra L, Cercignani M, Mastropasqua C, Torso M, Spanò B, Makovac E, Viola V, Giulietti G, Marra C, Caltagirone C et al (2016) Longitudinal changes in functional brain connectivity predicts conversion to Alzheimer's disease. J Alzheimers Dis 51(2):377-389

20. Ambastha AK (2015) Neuroanatomical characterization of Alzheimer's disease using deep learning. National University of Singapore, Singapore

21. Cuingnet R, Gerardin E, Tessieras J, Auzias G, Lehéricy S, Habert MO, Chupin M, Benali H, Colliot O, Initiative ADN et al (2011) Automatic classification of patients with Alzheimer's disease from structural MRI: a comparison of ten methods using the ADNI database. Neuroimage 56(2):766-781

22. Davatzikos C, Bhatt P, Shaw LM, Batmanghelich KN, Trojanowski JQ (2011) Prediction of $\mathrm{MCl}$ to $\mathrm{AD}$ conversion, via MRI, CSF biomarkers, and pattern classification. Neurobiol Aging 32(12):2322-e19

23. Suk HI, Lee SW, Shen D, Initiative ADN et al (2014) Hierarchical feature representation and multimodal fusion with deep learning for $A D / M C$ diagnosis. Neurolmage 101:569-582

24. Wee CY, Yap PT, Li W, Denny K, Browndyke JN, Potter GG, WelshBohmer KA, Wang L, Shen D (2011) Enriched white matter connectivity networks for accurate identification of $\mathrm{MCl}$ patients. Neuroimage 54(3):1812-1822

25. Zhang D, Shen D, Initiative ADN et al (2012) Predicting future clinical changes of $\mathrm{MCl}$ patients using longitudinal and multimodal biomarkers. PLoS ONE 7(3):e33182

26. Zhou L, Wang Y, Li Y, Yap PT, Shen D, ADNI, A.D.N.I. et al. (2011) Hierarchical anatomical brain networks for $\mathrm{MCl}$ prediction: revisiting volumetric measures. PLoS ONE 6(7):e21935

27. Greicius MD, Srivastava G, Reiss AL, Menon V (2004) Default-mode network activity distinguishes Alzheimer's disease from healthy aging: evidence from functional MRI. Proc Nat Acad Sci USA 101(13):4637-4642

28. Suk HI, Wee CY, Shen D (2013) Discriminative group sparse representation for mild cognitive impairment classification. In: International workshop on machine learning in medical imaging. Springer, pp 131-138

29. Gray KR, Wolz R, Heckemann RA, Aljabar P, Hammers A, Rueckert D, Initiative ADN et al (2012) Multi-region analysis of longitudinal FDG-PET for the classification of Alzheimer's disease. Neurolmage 60(1):221-229

30. Nordberg A, Rinne JO, Kadir A, Långström B (2010) The use of PET in Alzheimer disease. Nat Rev Neurol 6(2):78

31. Chen YJ, Deutsch G, Satya R, Liu HG, Mountz JM (2013) A semiquantitative method for correlating brain disease groups with normal controls using spect: Alzheimer's disease versus vascular dementia. Comput Med Imaging Graph 37(1):40-47

32. Górriz J, Segovia F, Ramírez J, Lassl A, Salas-Gonzalez D (2011) GMM based spect image classification for the diagnosis of Alzheimer's disease. Appl Soft Comput 11(2):2313-2325

33. Hanyu H, Sato T, Hirao K, Kanetaka H, Iwamoto T, Koizumi K (2010) The progression of cognitive deterioration and regional cerebral blood flow patterns in Alzheimer's disease: a longitudinal spect study. J Neurol Sci 290(1):96-101

34. Graña M, Termenon M, Savio A, Gonzalez-Pinto A, Echeveste J, Pérez J, Besga A (2011) Computer aided diagnosis system for Alzheimer disease using brain diffusion tensor imaging features selected by Pearson's correlation. Neurosci Lett 502(3):225-229

35. Lee W, Park B, Han K (2013) Classification of diffusion tensor images for the early detection of Alzheimer's disease. Comput Biol Med 43(10):1313-1320

36. Cui Y, Liu B, Luo S, Zhen X, Fan M, Liu T, Zhu W, Park M, Jiang T, Jin JS et al (2011) Identification of conversion from mild cognitive impairment to Alzheimer's disease using multivariate predictors. PLOS ONE 6(7):e21896

37. Fan Y, Rao H, Hurt H, Giannetta J, Korczykowski M, Shera D, Avants BB, Gee JC, Wang J, Shen D (2007) Multivariate examination of brain abnormality using both structural and functional MRI. Neurolmage 36(4):1189-1199

38. Hinrichs $C$, Singh V, Xu G, Johnson SC, Initiative ADN et al (2011) Predictive markers for $A D$ in a multi-modality framework: an analysis of $\mathrm{MCl}$ progression in the ADNI population. Neuroimage 55(2):574-589

39. Lu D, Popuri K, Ding W, Balachandar R, Beg MF (2017) Multimodal and multiscale deep neural networks for the early diagnosis of Alzheimer's disease using structural MR and FDG-PET images. arXiv:1710.04782

40. Perrin RJ, Fagan AM, Holtzman DM (2009) Multimodal techniques for diagnosis and prognosis of Alzheimer's disease. Nature 461(7266):916

41. Shi J, Zheng X, LiY, Zhang Q, Ying S (2018) Multimodal neuroimaging feature learning with multimodal stacked deep polynomial networks for diagnosis of Alzheimer's disease. IEEE J Biomed Health Inf 22(1):173-183

42. Suk HI, Shen D (2013) Deep learning-based feature representation for $\mathrm{ad} / \mathrm{MCl}$ classification. In: International conference on medical image computing and computer-assisted intervention. Springer, pp 583-590

43. Walhovd K, Fjell A, Brewer J, McEvoy L, Fennema-Notestine C, Hagler D, Jennings R, Karow D, Dale A, Initiative ADN et al (2010) Combining MR imaging, positron-emission tomography, and CSF biomarkers in the diagnosis and prognosis of Alzheimer disease. Am J Neuroradiol 31(2):347-354

44. Westman E, Muehlboeck JS, Simmons A (2012) Combining MRI and CSF measures for classification of Alzheimer's disease and prediction of mild cognitive impairment conversion. Neuroimage 62(1):229-238

45. Yuan L, Wang Y, Thompson PM, Narayan VA, Ye J, Initiative ADN et al (2012) Multi-source feature learning for joint analysis of incomplete multiple heterogeneous neuroimaging data. Neurolmage 61(3):622-632 
46. Zhang D, Shen D, Initiative ADN et al (2012) Multi-modal multi-task learning for joint prediction of multiple regression and classification variables in Alzheimer's disease. Neurolmage 59(2):895-907

47. Zhang D, Wang Y, Zhou L, Yuan H, Shen D, Initiative ADN et al (2011) Multimodal classification of Alzheimer's disease and mild cognitive impairment. Neuroimage 55(3):856-867

48. Ashburner J, Friston KJ (2000) Voxel-based morphometry - the methods. Neuroimage 11(6):805-821

49. Baron J, Chetelat G, Desgranges B, Perchey G, Landeau B, De La Sayette V Eustache $F(2001)$ In vivo mapping of gray matter loss with voxel-based morphometry in mild Alzheimer's disease. Neuroimage 14(2):298-309

50. Klöppel S, Stonnington CM, Chu C, Draganski B, Scahill RI, Rohrer JD, Fox NC, Jack CR Jr, Ashburner J, Frackowiak RS (2008) Automatic classification of MR scans in Alzheimer's disease. Brain 131(3):681-689

51. Maguire EA, Gadian DG, Johnsrude IS, Good CD, Ashburner J, Frackowiak RS, Frith CD (2000) Navigation-related structural change in the hippocampi of taxi drivers. Proc Natl Acad Sci 97(8):4398-4403

52. Friedman J, Hastie T, Tibshirani R (2001) The elements of statistical learning, vol 1. Springer, New York

53. Davatzikos C, Genc A, Xu D, Resnick SM (2001) Voxel-based morphometry using the RAVENS maps: methods and validation using simulated longitudinal atrophy. Neurolmage 14(6):1361-1369

54. Kohannim O, Hua X, Hibar DP, Lee S, Chou YY, Toga AW, Jack CR, Weiner MW, Thompson PM (2010) Boosting power for clinical trials using classifiers based on multiple biomarkers. Neurobiol Aging 31(8):1429-1442

55. Walhovd K, Fjell A, Brewer J, McEvoy L, Fennema-Notestine C, Hagler D, Jennings R, Karow D, Dale A (2010) the Alzheimerls disease neuroimaging initiative: combining MR imaging, positron-emission tomography, and CSF biomarkers in the diagnosis and prognosis of Alzheimer disease. Am J Neuroradiol 31:347-354

56. Zhang J, Liu M, An L, Gao Y, Shen D (2017) Alzheimer's disease diagnosis using landmark-based features from longitudinal structural MR images. IEEE J Biomed Health Inf 21(6):1607-1616

57. Atiya M, Hyman BT, Albert MS, Killiany R (2003) Structural magnetic resonance imaging in established and prodromal Alzheimer disease: a review. Alzheimer Dis Assoc Disord 17(3):177-195

58. Dubois B, Chupin M, Hampel H, Lista S, Cavedo E, Croisile B, Tisserand GL, Touchon J, Bonafe A, Ousset PJ et al (2015) Donepezil decreases annual rate of hippocampal atrophy in suspected prodromal Alzheimer's disease. Alzheimer's dement J Alzheimer's Assoc 11(9):1041-1049

59. Jack CR, Petersen RC, Xu YC, O'Brien PC, Smith GE, Ivnik RJ, Boeve BF, Waring SC, Tangalos EG, Kokmen E (1999) Prediction of AD with MRIbased hippocampal volume in mild cognitive impairment. Neurology 52(7):1397-1397

60. Lötjönen J, Wolz R, Koikkalainen J, Julkunen $V$, Thurfjell L, Lundqvist R, Waldemar G, Soininen H, Rueckert D, Initiative ADN et al (2011) Fast and robust extraction of hippocampus from MR images for diagnostics of Alzheimer's disease. Neuroimage 56(1):185-196

61. Coupé P, Eskildsen SF, Manjón JV, Fonov VS, Pruessner JC, Allard M, Collins DL, Initiative ADN et al (2012) Scoring by nonlocal image patch estimator for early detection of Alzheimer's disease. Neurolmage Clin 1(1):141-152

62. Gupta A, Ayhan M, Maida A (2013) Natural image bases to represent neuroimaging data. In: International conference on machine learning. pp 987-994

63. Liu M, Zhang D, Shen D (2014) Hierarchical fusion of features and classifier decisions for Alzheimer's disease diagnosis. Hum Brain Mapp 35(4):1305-1319

64. Liu M, Zhang D, Shen D, Initiative ADN et al (2012) Ensemble sparse classification of Alzheimer's disease. Neurolmage 60(2):1106-1116

65. Payan A, Montana G (2015) Predicting Alzheimer's disease: a neuroimaging study with 3D convolutional neural networks. arXiv:1502.02506

66. Wu G, Kim M, Sanroma G, Wang Q, Munsell BC, Shen D, Initiative ADN et al (2015) Hierarchical multi-atlas label fusion with multi-scale feature representation and label-specific patch partition. Neurolmage 106:34-46

67. Arvesen E (2015) Automatic classification of Alzheimer's disease from structural MRI. Master's thesis

68. Vemuri P, Gunter JL, Senjem ML, Whitwell JL, Kantarci K, Knopman DS, Boeve BF, Petersen RC, Jack CR (2008) Alzheimer's disease diagnosis in individual subjects using structural MR images: validation studies. Neuroimage 39(3):1186-1197

69. Gray KR (2012) Machine learning for image-based classification of Alzheimer's disease. Ph.D. thesis, Imperial College London

70. Er A, Varma S, Paul V (2017) Classification of brain MR images using texture feature extraction. Int J Comput Sci Eng 5(5):1722-1729

71. Morra JH, Tu Z, Apostolova LG, Green AE, Toga AW, Thompson PM (2010) Comparison of adaboost and support vector machines for detecting Alzheimer's disease through automated hippocampal segmentation. IEEE Trans Med Imaging 29(1):30

72. Liu M, Zhang J, Adeli E, Shen D (2018) Landmark-based deep multiinstance learning for brain disease diagnosis. Med Image Anal 43:157-168

73. Brosch T, Tam R, Initiative ADN et al (2013) Manifold learning of brain MRIs by deep learning. In: International conference on medical image computing and computer-assisted intervention. Springer, pp 633-640

74. Hosseini-Asl E, Keynton R, El-Baz A (2016) Alzheimer's disease diagnostics by adaptation of 3D convolutional network. In: 2016 IEEE international conference on image processing (ICIP). IEEE, pp 126-130

75. Liu F, Shen C (2014) Learning deep convolutional features for MRI based Alzheimer's disease classification. arXiv:1404.3366

76. Liu S, Liu S, Cai W, Che H, Pujol S, Kikinis R, Feng D, Fulham MJ (2015) Multimodal neuroimaging feature learning for multiclass diagnosis of Alzheimer's disease. IEEE Trans Biomed Eng 62(4):1132-1140

77. Sarraf S, Tofighi G (2016) Classification of Alzheimer's disease using fMRI data and deep learning convolutional neural networks. arXiv:1603.08631

78. Suk HI, Lee SW, Shen D, Initiative ADN et al (2015) Latent feature representation with stacked auto-encoder for AD/MCI diagnosis. Brain Struct Funct 220(2):841-859

79. Suk HI, Shen D, Initiative ADN (2015) Deep learning in diagnosis of brain disorders. In: Recent progress in brain and cognitive engineering. Springer, pp 203-213

80. Cárdenas-Peña D, Collazos-Huertas D, Castellanos-Dominguez G (2016) Centered kernel alignment enhancing neural network pretraining for MRI-based dementia diagnosis. Comput Math Methods Med 2016;2016:9523849. https://doi.org/10.1155/2016/9523849

81. Islam J, Zhang Y (2017) A novel deep learning based multi-class classification method for Alzheimer's disease detection using brain MRI data. Springer, Cham, pp 213-222. https://doi.org/10.1007/978-3-319-70772 -3 _20

82. Krizhevsky A, Sutskever I, Hinton GE (2012) Imagenet classification with deep convolutional neural networks. In: Advances in neural information processing systems. pp 1097-1105

83. Ioffe S, Szegedy C (2015) Batch normalization: accelerating deep network training by reducing internal covariate shift. In: International conference on machine learning. pp 448-456

84. Huang G, Liu Z, van der Maaten L, Weinberger KQ (2017) Densely connected convolutional networks. In: Proceedings of the IEEE conference on computer vision and pattern recognition

85. Khan SH, Hayat M, Bennamoun M, Sohel FA, Togneri R (2017) Costsensitive learning of deep feature representations from imbalanced data. IEEE Trans Neural Netw Learn Syst. 2017. https://doi.org/10.1109/TNNLS .2017.2732482

86. Abadi M, Agarwal A, Barham P, Brevdo E, Chen Z, Citro C, Corrado GS, Davis A, Dean J, Devin M, Ghemawat S, Goodfellow I, Harp A, Irving G, Isard M, Jia Y, Jozefowicz R, Kaiser L, Kudlur M, Levenberg J, Mané D, Monga R, Moore S, Murray D, Olah C, Schuster M, Shlens J, Steiner B, Sutskever I, Talwar K, Tucker P, Vanhoucke V, Vasudevan V, Viégas F, Vinyals O, Warden P, Wattenberg M, Wicke M, Yu Y, Zheng X (2015) TensorFlow: Large-scale machine learning on heterogeneous systems. https://www. tensorflow.org/, software available from tensorflow.org

87. Chollet F et al (2015) Keras. https://github.com/keras-team/keras

88. Szegedy C, loffe S, Vanhoucke V, Alemi AA (2017) Inception-v4, inceptionresnet and the impact of residual connections on learning. In: AAAl. pp $4278-4284$

89. He K, Zhang X, Ren S, Sun J (2016) Deep residual learning for image recognition. In: Proceedings of the IEEE conference on computer vision and pattern recognition. pp 770-778 